\title{
Resection of Thumb Metacarpal Ewing Sarcoma and Primary Reconstruction with Non-Vascularized Osteoarticular Metatarsal Autograft
}

\author{
Ariff $M^{\mathrm{a}}{ }^{\mathrm{b}}$, Faisham Wla $\mathrm{l}^{\mathrm{a}}$ Krishnan Ja, Zulmi Wa \\ a Orthopaedic Oncology and Reconstruction Unit, Department of Orthopaedics, School of Medical Sciences, \\ Universiti Sains Malaysia (USM), Malaysia. \\ ${ }^{b}$ Musculoskeletal Oncology Unit, Department of Orthopaedics, Traumatology and Rehabilitation, Kulliyyah \\ (Faculty) of Medicine, International Islamic University Malaysia (IIUM), Malaysia.
}

\begin{abstract}
Ewing sarcoma is a primary bone malignancy that rarely occurs in the hand. Resection and reconstruction will usually result in reduced or loss of thumb function. We describe a case of successful transplantation of nonvascularized osteoarticular second metatarsal autograft following wide resection of Ewing sarcoma of first metacarpal in an 11-year-old girl. The capsule of the graft's metatarso-phalangeal joint was repaired to achieve mobility of the joint. This enabled preservation of thumb function by a relatively simple surgical technique. Detailed surgical procedures and excellence function after 2 years following surgery are described.
\end{abstract}

KEYWORDS: Ewing sarcoma, hand tumour, metacarpal bone, thumb, non-vascularized graft

\section{INTRODUCTION}

Ewing's sarcoma is a primary bone malignancy; common in both the axial and appendicular skeletons but originates rarely in the bones of the hand. ${ }^{1,2}$ Historically, treatment involves combination of chemotherapy and radiotherapy. Radiotherapy is indicated in cases with incomplete surgical excision or resistant to chemotherapy. ${ }^{2}$ Neo-adjuvant chemotherapy is advocated in combination with wide resection and has proven to increase the survival rates. ${ }^{3,4}$ Depending on the anatomic location, the role of surgical intervention remains limited. This report describes a case of Ewing's sarcoma of the first metacarpal bone in an otherwise healthy young girl.

\section{CASE REPORT}

An 11-year-old, right-hand dominant girl presented with a painful swelling over her right hand for duration of 6 months. The swelling initially started over the base of her right thumb and gradually increased in size.
She could barely use her right hand for daily chores due to pain on movement. Examination revealed a firm mass over the thenar aspect of her right hand. The swelling was slightly tender involving the entire first metacarpal with thenar muscle extension. The metacarpo-phalangeal joint movement of the thumb was restricted due to pain. Conventional radiograph showed a dense, sclerotic, irregular lesion involving the metaphyseal and diaphyseal regions of the first metacarpal bone. There was also periosteal reaction noted (Figure 1 \& 2 ). Local MRI staging showed involvement of the whole first metacarpal bone and its marrow, with soft tissue extension to the adductor and flexor pollicis. The proximal and distal joints, as well as the long tendons to the thumb were free from infiltration. Further staging with computed tomography (CT) thorax revealed solitary lung nodule at the right lower zone of $0.5 \mathrm{~cm}$. Bone scan showed hot spot over the first metacarpal without other bone involvement.
Corresponding author:

Dr. Mohd Ariff Sharifudin

Musculoskeletal Oncology Unit,

Department of Orthopaedics, Traumatology

and Rehabilitation,

Kulliyyah (Faculty) of Medicine,

International Islamic

University Malaysia,Jalan Hospital,

25150 Kuantan, Pahang, Malaysia.

Tel: $\quad+6095706131 / 6145$

Fax: $\quad+6095144451$

Email: ariffs@iium.edu.my/mdariffs@hotmail.com 


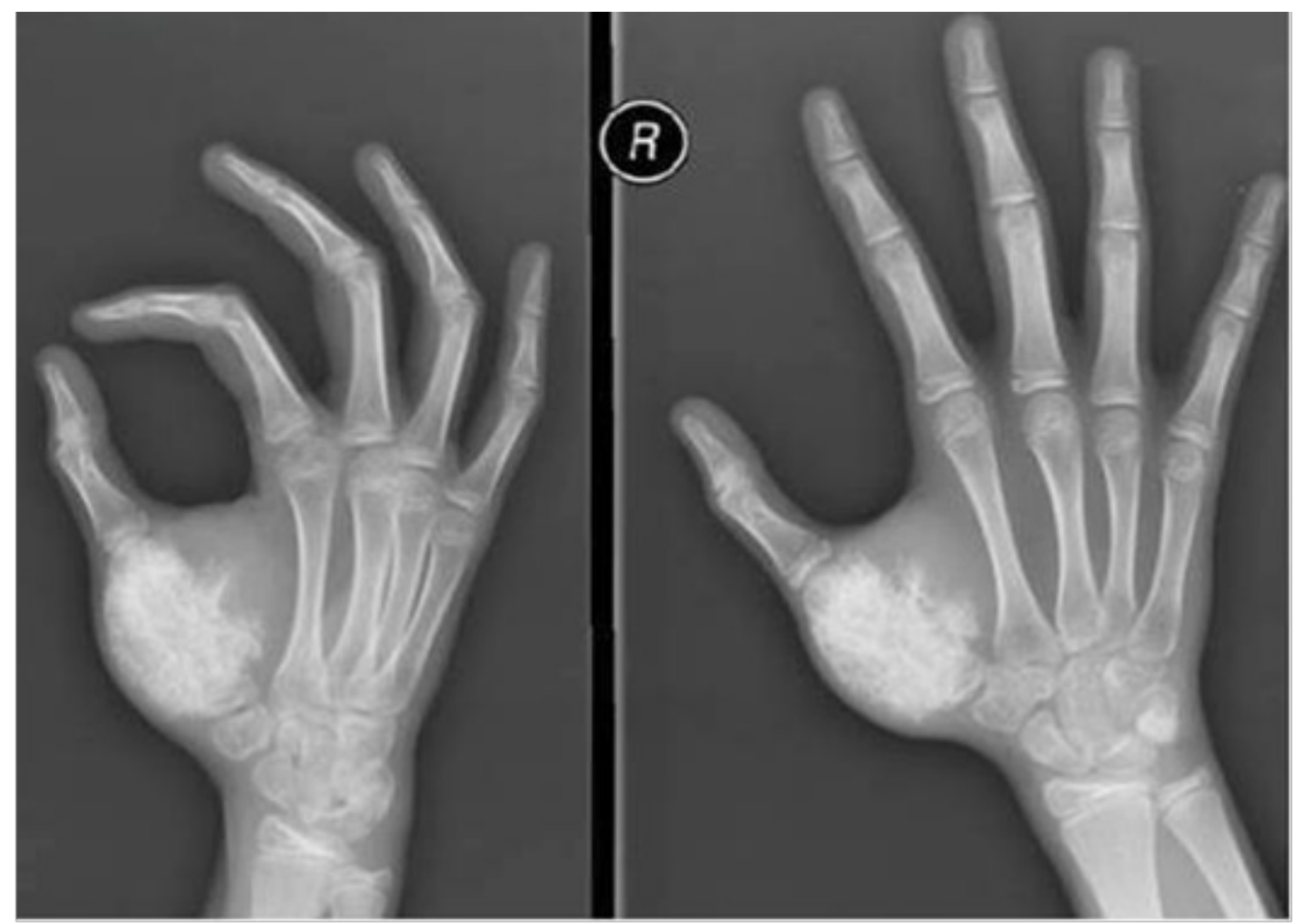

Figure 1. Plain radiographs (AP and oblique views) showing a destructive sclerotic bone lesion involving the entire first metacarpal with 'sunburst' periosteal reaction and soft tissue mass.

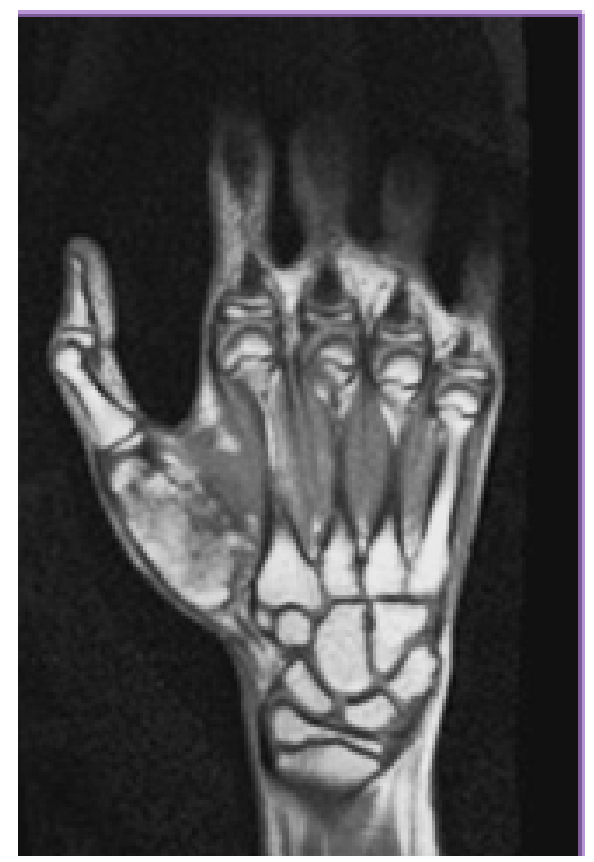

Figure 2. MRI T1 weighted showing a hyperintense lesion with involvement of the whole first metacarpal bone and its marrow, with soft tissue extension to the adductor and flexor pollicis.

A core biopsy was taken and the results were consistent with Ewing's sarcoma. She had underwent 8 cycles of neo-adjuvant chemotherapy, which consists of four drugs; Vincristin, Ifosfamide, Doxorubicin and Etoposide. Repeated staging with CT thorax showed that the pulmonary nodule had regressed. Local MRI revealed good response in the metacarpal tumour size but no evidence of necrosis.

Resection of the first metacarpal was performed through radial extensile approach. The first metacarpal neurovascular structures were explored and preserved over the first web space. The adductor pollicis were removed widely over the attachment of the metacarpal bone. Flexor pollicis were removed in total with the bone. It was an intra-articular excision as both proximal and distal joint were freed from tumour. The remnant of distal proximal phalanges capsule was preserved for future reconstruction (Figure 3a to 3d). 

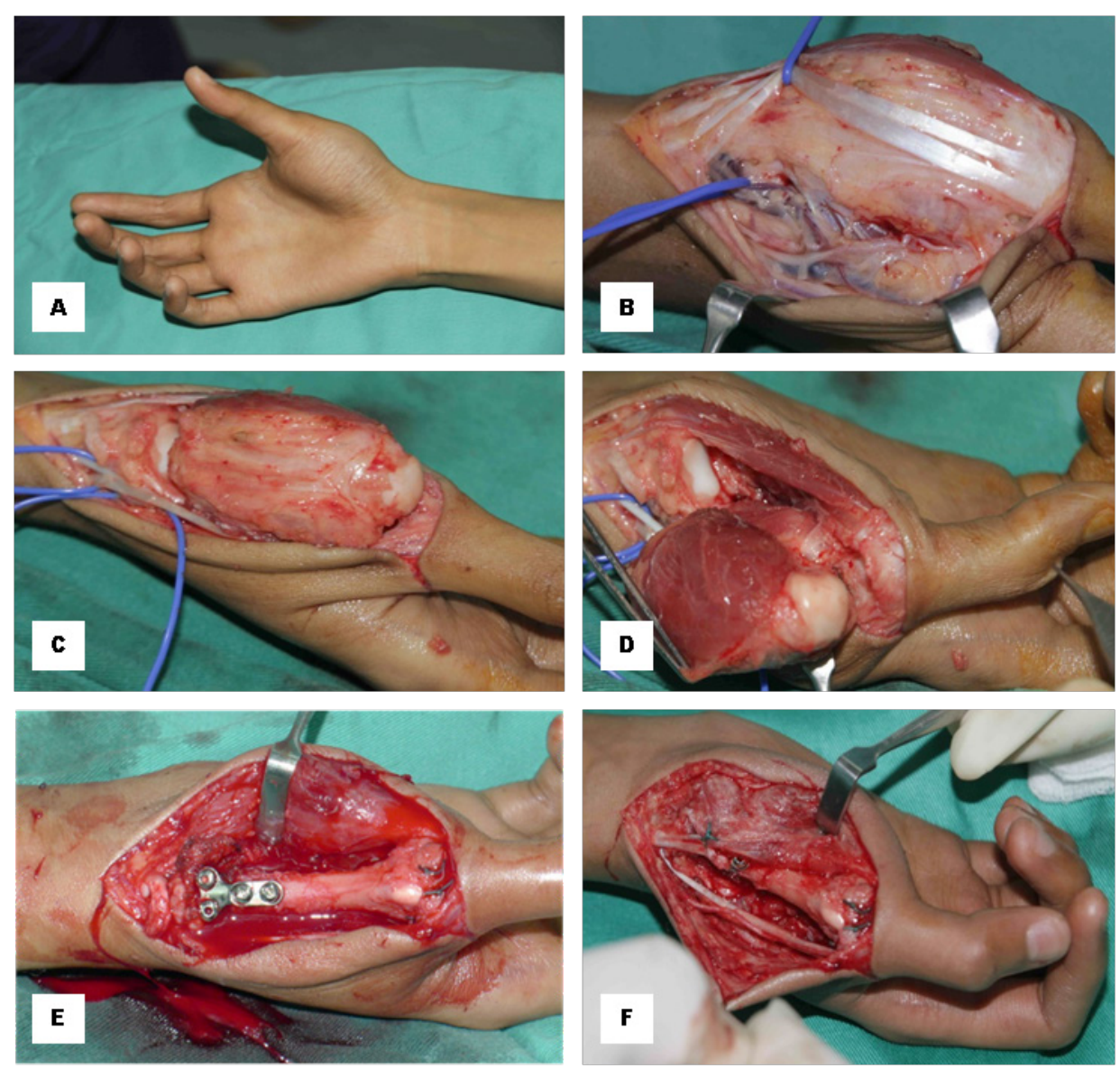

Figure 3. Surgical techniques of resection and primary reconstruction of the first metacarpal bone. (A) Preoperation. (B,C) Exploration and preservation of the neurovascular structures and the extensor pollicis tendons. (D) Intra-articular wide resection with partial preservation of the distal proximal phalanges capsule. (E,F) Thumb reconstruction utilizing second metatarsal bone; the metatarso-phalangeal joint capsule was reconstructed circumferentially with ethibond suture, and the metatarsal bone was fused with the trapezium with a mini Tplate.

The second metatarsal bone of her contralateral foot was resected through a dorsal approach. The capsule of the metatarso-phalangeal joint was taken with a cuff of $1 \mathrm{~cm}$ for reconstruction. An osteotomy was made of $4.5 \mathrm{~cm}$ to fit the metacarpal defect. The capsule was reconstructed circumferentially with ethibond suture, and the stability was assessed before the autogenous metatarsal graft was fixed to the trapezium and stabilized with a mini T-plate (Figure $3 e$ and $3 f$ ). The bone defect over the left foot were bridged with tricortical iliac bone graft and stabilized with intraosseous Kirschner wire through the joint for fusion. Post-operatively, her thumb was protected with thumb spica cast for 6 weeks followed by physiotherapy for thumb functions.

She completed another 6 cycles of adjuvant chemotherapy of similar regimes. After two years of follow-up, she was disease-free and the lung nodule completely regressed. She was able to write and regained a near normal function of her hand (Figure 4). Thorough hand assessment revealed active range of motion of first metacarpophalangeal joint at 10-45 degrees. There was no donor site morbidity at her left foot and left iliac crest as well. 

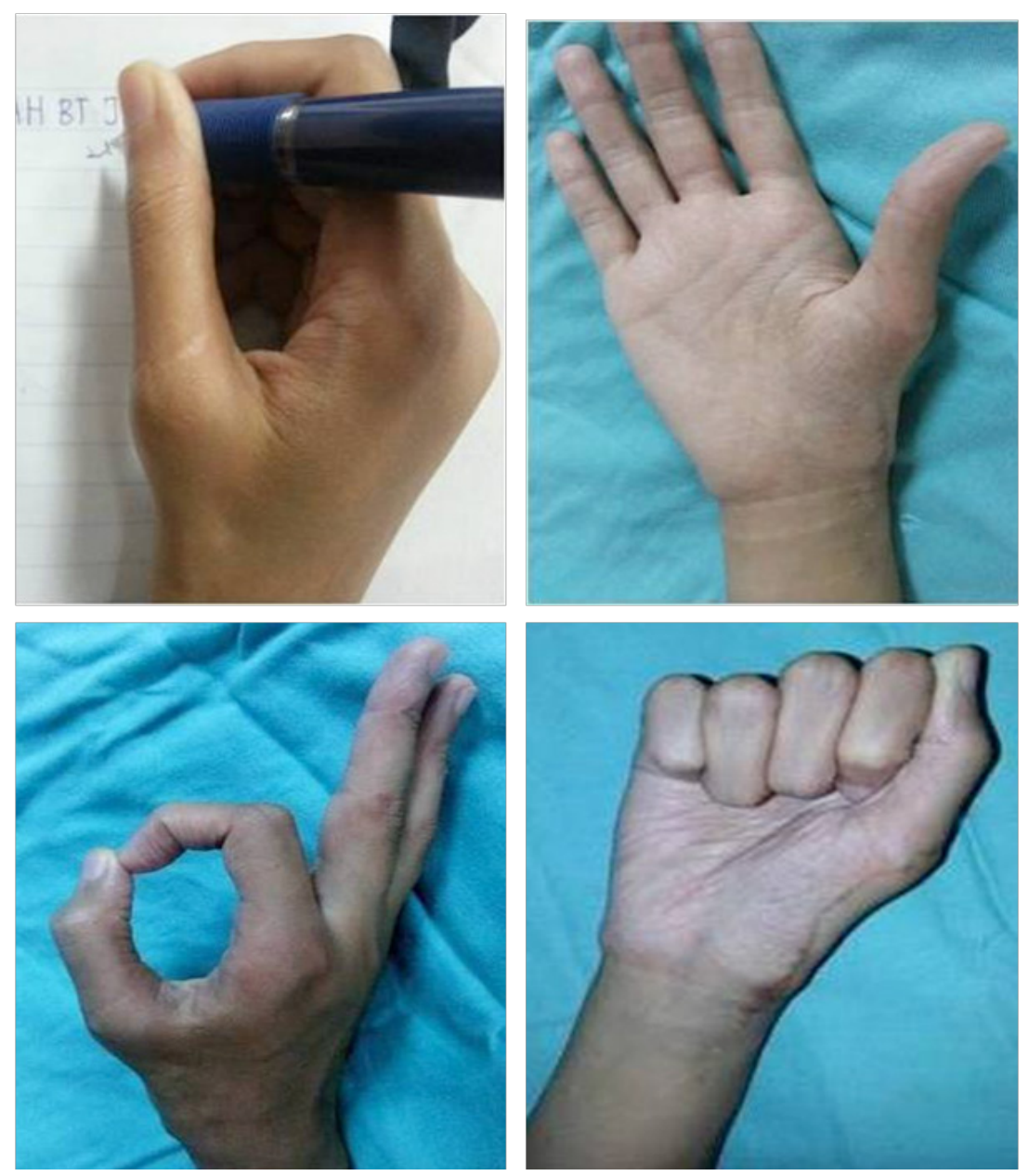

Figure 4. Normal hand function at two years' post-surgery.

\section{DISCUSSION}

Ewing sarcoma is the second most frequent primary bone malignancy of childhood and adolescence..$^{1,2}$ However, Ewing sarcoma of the hand is very rare and represents only $1 \%$ of all Ewing's sarcoma cases. ${ }^{2,4,5}$ The site of the tumour in the hand has a generally better prognosis compared to other sites, as survival is highest with involvement of distal bones of the extremities ${ }^{4,5}$. Pain and swelling of the affected fingers are the commonest presentation. ${ }^{1,3}$ Thus, complex surgical approach and donor site morbidities to achieve long-term functional outcomes are justified.

Wide resection of tumours of the metacarpal is generally reconstructed with allograft or autologous bone graft. The commonly used grafts are iliac or fibular bone grafts for carpo-metacarpal and metacarpo-phalangeal joint fusion. Metacarpophalangeal joint fusion may affect the movement of the thumb and its main functions. Options of thumb metacarpal reconstruction include segment of radius of radial forearm flap, or microsurgical transfer of vascularized fibular graft. ${ }^{6,7,8}$ However, these types of reconstruction require fusion of both joints. The options are best for tumour with extensive soft tissue and muscle extension whereby remaining function is

Volume 14 Number 1, June 2015 expected to be compromised.

We opted for second metatarsal bone transfer to achieve mobility of the metacarpo-phalangeal joint and the size of matched between two joints. Fusion of carpo-metacarpal joint as described in previous cases caused minimal functional loss. Reported cases of hand tumours reconstructed with metatarsal graft following resection were mostly adult patients with benign tumours such as giant cell tumours, aneurysmal bone cyst, and fibrous dysplasia.7,8,9 Using a metatarsal bone provides a good joint surface for articular reconstruction and requires minimal refashioning of the graft. Metatarsal graft also gives a good cosmetic shape. Compared to an allograft, autogenous metatarsal graft would have a better chance of union with minimal loss of foot function. Metacarpo-phalangeal joint reconstruction provides adequate stability so no further fixation technique is required. The synovial membrane of the proximal phalanx will provide nutrition to the cartilage of the transferred metatarsal to ensure its survival. Osteoarticular ligamentous complex reconstruction following the metatarsal transfer is a relatively simple procedure that any general orthopaedic surgeon can do. ${ }^{9}$ 
Graft resorption had been the problem of auto-transplantation of non-vascularized bone graft. In most cases, vascularized bone transfer is much more elegant and will preserve the articular cartilage vascularity. It is certainly possible to perform a vascularized second metatarsal transfer to replace a metacarpal. The vascularized bone will not resorb as much and the articular perfusion will be maintained. However, such procedure will be technically demanding and time consuming. We postulated that healing in paediatric patients are much better than adults; hence, a non-vascularized graft is sufficient to achieve good outcomes. At two years follow-up, the patient achieved near normal functions of her dominant hand, with no donor sites morbidity (Figure 4).

\section{CONCLUSION}

Resection and reconstruction surgeries in the current case discussed are described as a relatively simple single-stage procedure. The early functional outcome of the thumb was excellence. This option should be considered in hand tumours involving paediatric population.

\section{REFERENCES}

1. Baccari S, Hamdi MF, Mabrouki Z, Daghfous M, Tarhouni L. Ewing's sarcoma of the finger: Report of two cases and literature review. Orthop Traumatol Surg Res 2012;98:233-7.

2. Anakwenze OA, Parker WL, Wold LE, Amrami KK, Amadio PC. Ewing's sarcoma of the hand. J Hand Surg Eur 2009;34:35-9.

3. Jerome TJ, Varghese M, Sankaran B. Ewing's sarcoma of the distal phalanx of the little finger. J Hand Surg Eur Vol 2008;33:81-2.

4. Jones MW. Ewing's sarcoma of the thumb. J Hand Surg Br 1993;18:356-7.

5. Kissane JM, Askin FB, Foulkes M, Stratton LB, Shirley SF. Ewing's sarcoma of bone: clinicopathologic aspects of 303 cases from the Intergroup Ewing's Sarcoma Study. Hum Pathol 1983; 14:773-9.

6. Garg P, Walia SK, Singh B, Walia JPS. Giant cell tumour of third metacarpal: a case report. $\mathrm{Pb}$ Journal of Orthopaedics 2010;12:27-9.

7. Rao GS, Keogh P, Webster H, Lunn PG, Burke FD. Aneurysmal bone cysts in the hand treated by free non-vascular transfer of metatarsal or proximal phalanx from the foot. J Hand Surg Eur Vol 1993; 18:736-41.

8. Verma RR, Paul A. Fibrous dysplasia of the fourth metacarpal: en-bloc resection and free metatarsal transfer. Orthopedics 2006; 29:371-2.

9. Maini L, Cheema GS, Yuvarajan P, Gautam VK. Free osteoarticular metatarsal transfer for giant cell tumor of metacarpal: a surgical technique. J Hand Microsurg 2011; 3:89-92. 